\title{
Coordinated Supervisory Control of Multi-Terminal HVDC Grids: a Model Predictive Control Approach
}

\author{
Lampros Papangelis, Student Member, IEEE, Marie-Sophie Debry, \\ Patrick Panciatici, Senior Member, IEEE, Thierry Van Cutsem, Fellow, IEEE
}

\begin{abstract}
A coordinated supervisory control scheme for future multi-terminal High-Voltage Direct-Current (HVDC) grids is proposed. The purpose is to supervise the grid and take appropriate actions to ensure power balance and prevent or remove voltage or current limit violations. First, using DC current and voltage measurements, the power references of the various Voltage Sources Converters (VSC) are updated according to participation factors. Next, the setpoints of the converters are smoothly adjusted to track those power references, while avoiding or correcting limit violations. The latter function resorts to Model Predictive Control and a sensitivity model of the system. The efficiency of the proposed scheme has been tested through dynamic simulations of a five-terminal HVDC grid interconnecting two asynchronous $\mathrm{AC}$ areas and a wind farm.
\end{abstract}

Index Terms-Multi-terminal HVDC grids, voltage source converter, corrective control, model predictive control

\section{NOMENCLATURE}

$\boldsymbol{P}, \boldsymbol{P}^{\text {set }} \quad$ VSC powers and corresponding setpoints.

$\boldsymbol{V}, \boldsymbol{V}^{\text {set }}$ VSC DC voltages and corresponding setpoints.

I DC branch currents.

$\boldsymbol{P}^{m} \quad$ VSC power measurements.

$\boldsymbol{V}^{m} \quad$ VSC DC voltage measurements

$\boldsymbol{I}^{m} \quad$ DC branch current measurements.

$\boldsymbol{K}_{V} \quad$ Diagonal matrix of VSC droop gains.

$N_{c}, N_{p} \quad$ Control and prediction horizons.

$N, N_{D} \quad$ No. of VSCs, no. of dispatchable VSCs.

$\boldsymbol{P}^{s c h}, \boldsymbol{P}^{\text {ref }}$ Scheduled and reference powers of VSCs.

$\rho_{i} \quad$ Participation factor of $i$-th VSC.

$\Delta \boldsymbol{P}^{\text {set }} \quad$ Changes of VSC power setpoints.

$\boldsymbol{V}_{\text {avg }}, \boldsymbol{V}_{\text {avg }}^{r e f}$ Average of VSC DC voltages and corresponding reference.

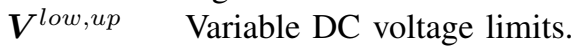

$\boldsymbol{V}^{\min , \max }$ Steady-state DC voltage limits.

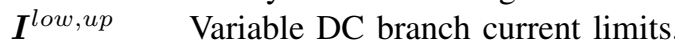

$\boldsymbol{I}^{\min , \max }$ Steady-state branch current limits.

$\boldsymbol{S}_{P} \quad$ Sensitivity matrix relating VSC DC voltages to VSC power setpoint changes.

$\boldsymbol{S}_{I} \quad$ Sensitivity matrix relating VSC power setpoint changes to DC branch currents.

Vectors and matrices are denoted with bold letters.

L. Papangelis is with the Dept. of Electrical Eng. and Computer Sc., University of Liège, Belgium. E-mail: 1.papangelis@ulg.ac.be.

M.-S. Debry and P. Panciatici are with the Research and Development Dept. of RTE (the French transmission system operator), Versailles, France. E-mails: marie-sophie.debry@rte-france.com, patrick.panciatici@rte-france.com.

T. Van Cutsem is with the Fund for Scientific Research (FNRS) at the Dept. of Electrical Eng. and Computer Sc., University of Liège, Belgium. E-mail: t.vancutsem@ulg.ac.be.

\section{INTRODUCTION}

The increasing need for bulk power transfer over long distances driven by the shift to renewable energy production and the gradual replacement of conventional power plants has led to the development of HVDC systems using the Voltage Source Converter (VSC) technology. The next step, after solutions for DC fault clearing have been implemented, is the extension to Multi-terminal DC (MTDC) systems [1].

The secure and robust operation of an MTDC grid poses several challenges [1]. An important one is controlling the power balance of the MTDC grid despite its relatively small energy storage capacity. Various methods have been proposed to tackle this issue. Most of them resort to the DC voltage droop technique to quickly correct a power imbalance at MTDC grid level and, hence, stabilize DC voltages [2].

However, these local controls alone cannot remove systemwide violations, such as DC branch overloads, or steer the MTDC grid towards a desired operating point. For this reason, other works are devoted to updating the power and/or DC voltage setpoints transmitted to the VSCs. To this purpose, as a higher-level control, the authors of [3] and [4] propose an Optimal Power Flow (OPF) to determine and send setpoints to the VSCs at regular time intervals, with the objective of minimizing losses. The more recent work in [5] computes the power and voltage setpoints by solving a multi-objective optimization. The coordinated control detailed in [6] uses repeated DC power flow computations to update the voltage and power setpoints at regular time intervals. Similarly, a method is proposed in [7], [8] to estimate the setpoint corrections to be sent to the VSCs so that the power flows through selected AC/DC terminals are restored. A comparison of various redispatch schemes is offered in [9] with the same objective of correcting the voltage offset left by voltage droop control after a disturbance. The ability of the various schemes to track the desired power setpoints is also evaluated.

Reference [10] proposes a three-level control structure inspired of AC frequency control practice. Primary control consists of a simple current-based droop scheme. The secondary level involves a slow Proportional-Integral (PI) control to reset the VSC powers to their reference values, and a redispatch scheme updating the power references at regular time intervals. Reference is made to OPF for tertiary control.

Coming back to DC voltage control, several alternatives have been also proposed. The work in [11] relies on multiple "master" converters whose voltage setpoints are determined by (centrally) solving a security-constrained OPF. The latter 
minimizes MTDC grid losses while keeping DC voltages within limits after the outage of a VSC. Reference [12] introduces the pilot voltage droop concept, in which a common DC voltage is communicated to all converters for them to share the powers more efficiently. In addition, two methods are used for power setpoint tracking, namely a simple PI controller and a setpoint redispatching by a central entity. A different approach is followed in [13], where fast communication is used to match at each time step the sum of DC currents injected by wind farms with the sum of DC currents of the grid-side converters.

This paper proposes a scheme for MTDC grid supervisory control. It is coordinated, i.e. it receives measurements from the MTDC system and sends back setpoints to the AC/DC converters. Furthermore, it satisfies the following requirements:

1) accommodate the varying power injections by renewable sources;

2) be robust with respect to model inaccuracies as well as disturbances, such as outages of AC/DC converters or DC cables/lines;

3) prevent or correct DC voltage and current violations;

4) smoothly drive the system from the current to a desired operating point;

5) avoid excessive impact on the adjacent AC systems and provide additional services (such as frequency support).

6) avoid extensive communication between controllers.

Optimization-based approaches are the most appealing owing to their ability to handle constraints. However, most optimization methods proposed in the literature have been devised to operate in open loop. Hence, they cannot account for model inaccuracies, component failures, etc. This was the authors' main motivation for resorting to Model Predictive Control (MPC) [14], [15].

Simply stated, MPC consists of computing a sequence of control changes which minimizes a multi-time-step objective and satisfies constraints in the future [14]. This optimization relies on a model of the system evolution. At a given discrete time $k$, using the latest available measurements, the controller computes a sequence of optimal control actions to be applied from $k$ to $k+N_{c}-1$, so that the system meets a desired target at $k+N_{p}$, where $N_{p} \geq N_{c} . N_{c}$ and $N_{p}$ are referred to as control and prediction horizons, respectively. Out of this sequence, only the first component is applied. Then, at the next time instant $k+1$, the procedure is repeated for the updated control and prediction horizons, using the newly received measurements. This yields a closed-loop behavior, that can account for unexpected behavior and approximation of the system model.

MPC has been already envisaged in HVDC systems, for fast power tracking of a VSC [16], electromechanical oscillation damping [17], or secondary frequency control [18]. Closer to this paper, MPC has been proposed for MTDC grid control in [19]. However, that work is more economy oriented with a time horizon in the order of hours, and does not address system control in the presence of limit violation or after an unforeseen outage. The transition between operating points is not considered either. Instead, the MPC-based scheme

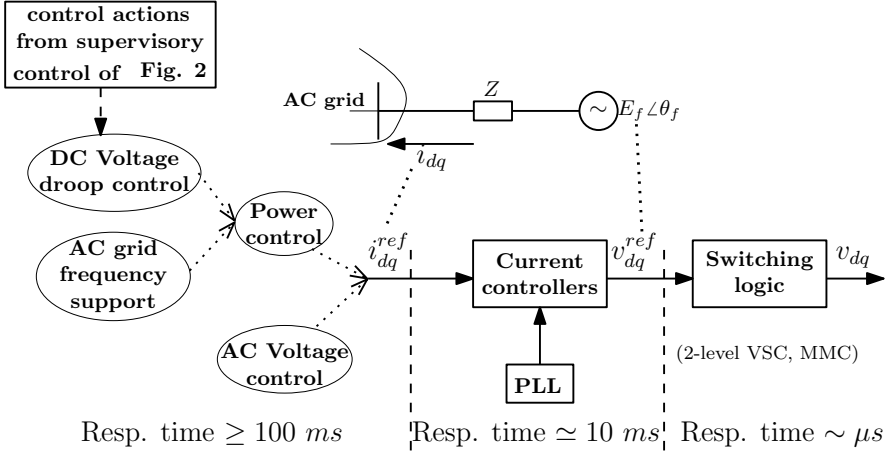

Fig. 1. Simplified diagram of the VSC control structure

proposed in this paper is aimed at steering the VSCs with a response time ranging from seconds up to a couple of minutes.

The rest of the paper is organized as follows. Section II recalls some basics of VSC control. Section III details the proposed control. Simulation results are presented in Section IV. Conclusions are offered in Section V.

\section{OVERVIEW OF VSC CONTROL BASICS}

This section briefly recalls some basics of VSC control with emphasis on the DC voltage droop technique.

As illustrated in Fig. 1, at fundamental frequency, a VSC is seen from the AC network as a voltage source behind an impedance. The active and reactive powers exchanged with the AC grid are controlled through a cascade of feedback loops with well decoupled time responses [6], [20]. The control structure is also sketched. The switching logic is the fastest; it depends on the type of converter, i.e. two/three-level VSC or Modular Multilevel Converter. The next level consists of the Phase Lock Loop (PLL) and the current controllers. The former synchronizes the VSC with the AC grid, while the latter adjust the VSC voltage source to bring the active and reactive currents to their references. The outer controllers provide these references based on various objectives, an indicative subset of which being shown in Fig. 1. The setpoints of these outer controllers can in turn be modified by a supervisory controller acting at grid level. This is further explained in Section III.

Controlling the DC voltages is of crucial concern for the correct operation of an MTDC grid. Indeed, power imbalances must be rapidly corrected, given the relatively small amount of energy stored in DC capacitors. Several methods have been proposed to this purpose. The DC voltage droop technique has received significant attention [2] and has been adopted in this work. Inspired of AC frequency control practice, it allows a power imbalance to be shared by multiple converters while ensuring redundancy against the outage of one of them. In a droop-controlled MTDC grid some of the VSCs obey a $P-V$ characteristic defined by a power setpoint $P^{s e t}$, a voltage setpoint $V^{\text {set }}$ and a droop gain $K_{V}$. In steady state the VSC power $P$ is linked to the DC voltage $V$ through:

$$
P=P^{s e t}-K_{V}\left(V-V^{s e t}\right)
$$

where a positive power corresponds to rectifier operation. Following a power deficit of the MTDC grid, the DC voltage 


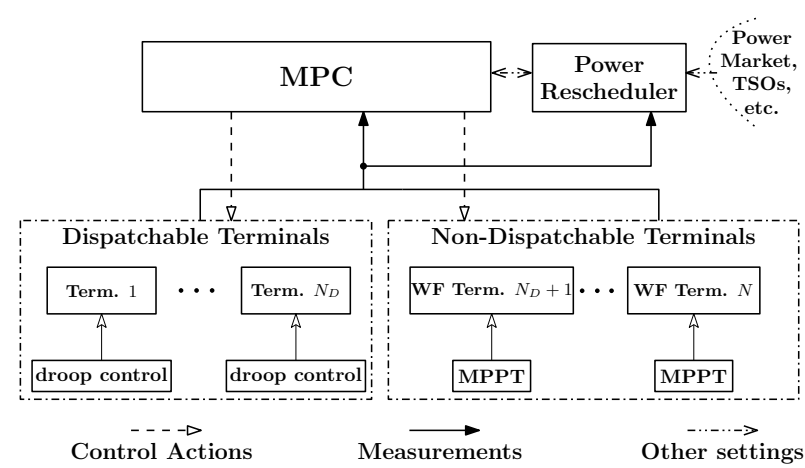

Fig. 2. Overall control structure

starts decreasing with the effect that the VSC increases its power injected into the DC grid until the balance is restored.

\section{Proposed CEnTRALized CONTROL}

\section{A. Overall controller description}

As already mentioned, the twofold purpose of the proposed centralized control is: (i) to smoothly drive the MTDC system from one operating point to another, and (ii) correct DC node voltage and branch current violations.

Following a similar categorization to the one in [5], the MTDC grid is assumed to be connected through dispatchable and non-dispatchable VSCs, respectively. Dispatchable VSCs are assigned a $P-V$ droop characteristic according to Eq. (1). They either participate in DC voltage control or keep their power to a constant value (i.e. $K_{V}=0$ ) as specified, for instance, by market agreements. They are generally connected to strong AC areas. The non-dispatchable VSCs, on the other hand, have their power varied by external factors. For instance, a VSC connecting an offshore Wind Farm (WF) to the MTDC grid is considered non-dispatchable, since it transfers the power collected by the WF, usually operating in Maximum Power Point Tracking (MPPT) mode. Hence, they do not participate in DC voltage control (i.e. $K_{V}=0$ ).

Supervisory control should act primarily on dispatchable terminals. Adjustments of non-dispatchable terminals (e.g. to decrease the infeed of a WF [21], [22]) should be reserved for cases where a limit violation cannot by removed by dispatchable terminals only.

The overall control structure is shown in Fig. 2. The lower layer includes a total of $N$ terminals, out of which $N_{D}$ (resp. $N-N_{D}$ ) are dispatchable (resp. non-dispatchable). The upper layer consists of a Power Rescheduler and the MPCbased controller, both acting at the same discrete times. Their respective functions are explained in the next sub-sections.

\section{B. Power Rescheduler: updating the VSC reference powers}

The Power Rescheduler calculates the power references $\boldsymbol{P}^{r e f}$, which are the desired VSC power flows. For all dispatchable VSCs, it receives a power schedule $\boldsymbol{P}^{s c h}$, which may be provided by the market operator, by AC transmission system operators, etc. This schedule is assumed to be updated only infrequently. In the meantime, to cope with the variability
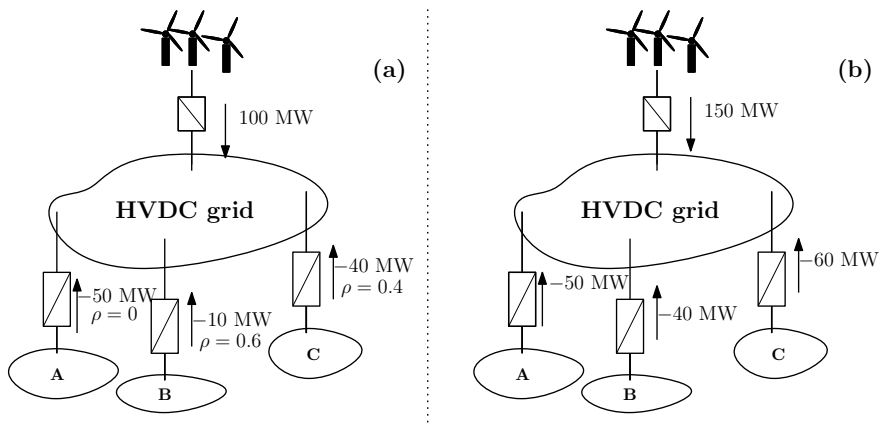

Fig. 3. VSC power references: (a) in base case; (b) after a $50 \mathrm{MW}$ increase of WF power

of the non-dispatchable VSC powers, it is necessary to adjust the power references of the dispatchable VSCs. This can be achieved through a variety of procedures. One simple option, considered in this work, is to use VSC participation factors $\rho_{i}$, as explained hereafter.

First, for non-dispatchable VSCs, the reference power is taken equal to the last available measurement, i.e.

$$
P_{i}^{r e f}=P_{i}^{m} \quad i=N_{D}+1, \ldots, N
$$

which amounts to considering that the non-dispatchable VSC powers are not going to change. A forecasted value (of the WF powers) could be used instead but, in view of the short horizon covered by the controller, this may not be very relevant.

Next, the power references of the dispatchable VSCs are adjusted according to $\left(i=1, \ldots, N_{D}\right)$ :

$$
\begin{gathered}
P_{i}^{r e f}=P_{i}^{s c h}-\rho_{i}\left(\sum_{l=1}^{N_{D}} P_{l}^{s c h}+\sum_{l=N_{D}+1}^{N} P_{l}^{r e f}\right) \\
\text { where: } \quad \sum_{i=1}^{N_{D}} \rho_{i}=1 \quad \text { and } \quad \rho_{i} \geq 0 .
\end{gathered}
$$

If the MTDC grid was lossless, the non-dispatchable powers constant and their measurements $P_{i}^{m}$ infinitely accurate, the parenthesis in Eq. (3) would be zero. In a real situation, it will differ from zero due to grid losses, measurement noise and variations of the non-dispatchable powers. While the latter are accounted for through Eq. (2), losses and measurement noise are not considered in Eq. (3). Losses could be accounted through some power flow computation, but MPC easily accommodates the above simplification.

The illustrative example of a hypothetical system is given in Fig. 3. Figure 3.(a) shows the initial schedule (neglecting grid losses) provided to the Power Rescheduler, corresponding to a WF power of $100 \mathrm{MW}$. It states that $50 \mathrm{MW}$ are injected in area $\mathrm{A}, 10 \mathrm{MW}$ in area $\mathrm{B}$ and the rest in area $\mathrm{C}$. The participation factors are such that the injection in area $\mathrm{A}$ does not deviate from $50 \mathrm{MW}$, while $60 \%$ of the WF power change is covered by area B and the rest by area C. Figure 3.(b) shows the power references given by Eq. (3) after an increase of the WF power by $50 \mathrm{MW}$. 


\section{MPC: constrained optimization problem}

The objective of the MPC block in Fig. 2 is to smoothly steer the VSCs to the reference powers given by the Power Rescheduler while obeying the following constraints:

- lower and upper limits on DC node voltages;

- lower and upper limits on the power of each VSC;

- limit on the rate of change of each VSC power;

- upper limit on each DC branch current, etc.

To this end, the controller receives measurements at regular time intervals. At time $k$ the following measurements are used:

$\boldsymbol{P}^{m}(k)$ the vector of VSC powers;

$\boldsymbol{V}^{m}(k)$ the vector of DC node voltages;

$\boldsymbol{I}^{m}(k)$ the vector of DC branch currents.

Based on these measurements, a reference trajectory [15] is defined with the objective of steering the VSC powers to the reference values $\boldsymbol{P}^{r e f}$ in $N_{c}$ control steps. That trajectory is linear and defined as follows $\left(j=1, \ldots, N_{c}\right)$ :

$$
\boldsymbol{P}^{r e f}(k+j)=\boldsymbol{P}^{m}(k)+\frac{j}{N_{c}}\left(\boldsymbol{P}^{r e f}-\boldsymbol{P}^{m}(k)\right) .
$$

The MPC objective consists of minimizing the deviations with respect to that reference trajectory:

$$
\begin{array}{r}
\min _{\boldsymbol{V}, \boldsymbol{I}, \boldsymbol{P}, \boldsymbol{\epsilon}, \boldsymbol{\zeta}, \Delta \boldsymbol{P}^{\text {set }}, V_{\text {avg }}} \sum_{j=1}^{N_{c}}\left\|\boldsymbol{P}^{r e f}(k+j)-\boldsymbol{P}(k+j)\right\|_{\boldsymbol{W}}^{2} \\
+v \sum_{j=1}^{N_{c}}\|\boldsymbol{\epsilon}(k+j)\|^{2}+q \sum_{j=1}^{N_{c}}\|\boldsymbol{\zeta}(k+j)\|^{2}
\end{array}
$$

where $\mathbf{W}$ is a diagonal weighting matrix assigned to the deviations of VSC powers $\boldsymbol{P}$ from their references $\boldsymbol{P}^{r e f}$. Nondispatchable terminals are assigned a weight $w_{N D}$ higher than the weight $w_{D}$ of dispatchable VSCs in order to resort to them when actions on dispatchable VSCs only are not sufficient. $\epsilon$ and $\zeta$ are vectors of slack variables used to relax constraints in case of infeasibility, with the respective weighting factors $v$ and $q$.

The minimization is subject to the following constraints: for $j=1, \ldots, N_{c}$ :

$$
\begin{gathered}
\boldsymbol{V}^{\text {low }}(k+j)-\boldsymbol{\epsilon}(k+j) \leq \boldsymbol{V}(k+j) \\
\leq \boldsymbol{V}^{\text {up }}(k+j)+\boldsymbol{\epsilon}(k+j) \\
\boldsymbol{P}^{\text {min }} \leq \boldsymbol{P}(k+j) \leq \boldsymbol{P}^{\text {max }} \\
\Delta \boldsymbol{P}^{\text {min }}-\boldsymbol{\zeta}(k+j) \leq \boldsymbol{P}(k+j)-\boldsymbol{P}(k+j-1) \\
\leq \Delta \boldsymbol{P}^{\text {max }}+\boldsymbol{\zeta}(k+j) \\
\boldsymbol{I}^{\text {low }}(k+j) \leq \boldsymbol{I}(k+j) \leq \boldsymbol{I}^{\text {up }}(k+j) \\
\boldsymbol{\epsilon}(k+j) \geq \mathbf{0}, \quad \boldsymbol{\zeta}(k+j) \geq \mathbf{0} \\
\Delta \boldsymbol{P}^{\text {set }}(k+j-1)=\boldsymbol{S}_{P}[\boldsymbol{V}(k+j)-\boldsymbol{V}(k+j-1)] \\
\boldsymbol{P}(k+j)=\boldsymbol{P}(k+j-1)+\Delta \boldsymbol{P}^{\text {set }}(k+j-1) \\
-\boldsymbol{K}_{V}[\boldsymbol{V}(k+j)-\boldsymbol{V}(k+j-1)] \\
\boldsymbol{I}(k+j)=\boldsymbol{I}(k+j-1)+\boldsymbol{S}_{I} \Delta \boldsymbol{P}^{\text {set }}(k+j-1) \\
V_{\text {avg }}(k+j)=V_{\text {avg }}(k)+\frac{j}{N_{c}}\left(V_{\text {avg }}^{\text {ref }}-V_{\text {avg }}(k)\right) .
\end{gathered}
$$

Constraint (7) sets the range of admissible DC voltages at the VSC buses. Any violation of these limits is heavily penalized in the objective function by setting the weighting factor $v$ to a large value. Note that $\boldsymbol{V}^{\text {low }}$ and $\boldsymbol{V}^{\text {up }}$ evolve with time, in order to bring the voltages progressively inside the desired range. Similarly, constraint (10) keeps the branch currents $\boldsymbol{I}$ between limits which vary with time for the same purpose. This variation is further detailed in Appendix A.

Constraint (8) relates to the minimum and maximum power of each VSC.

Constraint (9) stems from the AC networks. Too fast changes in the power injections into or from the $\mathrm{AC}$ areas could cause problems, such as unacceptable frequency or voltage deviations. Therefore, this constraint limits the power change of each VSC between two discrete time steps to $\Delta \boldsymbol{P}^{\min }$ and $\Delta \boldsymbol{P}^{\max }$. The slack variables $\zeta$ are also heavily penalized in the objective function by choosing a large value for the weighting factor $q$.

Equations (12), (13) and (14) are used to predict future values of voltages, powers and currents in response to the control changes $\Delta \boldsymbol{P}^{\text {set }}$, which obey:

$$
\boldsymbol{P}^{s e t}(k+j)=\boldsymbol{P}^{\text {set }}(k+j-1)+\Delta \boldsymbol{P}^{\text {set }}(k+j-1) .
$$

For uniformity the notation $\Delta P^{\text {set }}$ is also used for actions on the non-dispatchable terminals, although they are not assigned a setpoint $P^{s e t}$. Since the non-dispatchable terminals do not participate in DC voltage control, $\Delta P^{\text {set }}$ is actually the desired change in power $\Delta P$.

The prediction horizon is equal to the control horizon $N_{c}$. This transition model relies on sensitivity matrices, which is justified by the fast response of the power electronics based VSCs compared to the MPC sampling time, in the order of a few seconds. The derivation of those matrices is detailed in the next sub-section.

The above linear model is initialized at the last received measurements:

$$
\boldsymbol{V}(k)=\boldsymbol{V}^{m}(k) \quad \boldsymbol{P}(k)=\boldsymbol{P}^{m}(k) \quad \boldsymbol{I}(k)=\boldsymbol{I}^{m}(k) .
$$

The last constraint (15) is aimed at restoring the average DC voltage:

$$
V_{\text {avg }}(k+j)=\frac{1}{N} \sum_{i=1}^{N} V_{i}(k+j) \quad j=0, \ldots, N_{c}
$$

to a given reference $V_{a v g}^{r e f}$. The main motivation is that, in an MTDC system, no bus has its voltage tightly controlled; instead, DC voltages evolve according to the droop characteristic of Eq. (1) (where an infinite $K_{V}$ would be required to have constant voltage). Equation (15) prevents the voltage profile from somewhat "floating". Without this constraint, if the losses in the MTDC grid are underestimated by the Power Rescheduler when setting $\boldsymbol{P}^{\text {ref }}$, the MPC controller will progressively increase the DC voltages (until one of them hits its limit) in order to decrease the losses and, hence, the deviations from the reference powers. Conversely, if the MTDC grid losses are overestimated, the MTDC voltages will be pushed to their lower limits. $V_{a v g}^{r e f}$ can be simply set to 
the nominal DC voltage (i.e. to $1 \mathrm{pu}$ ) or it can be chosen to minimize the grid losses for a wide range of scenarios.

By keeping the DC voltages around the reference, Eq. (15) contributes to keeping voltages within their limits. However, controlling the average does not ensure that all voltages settle in the desired range. This is why the constraints (7) are also included in the formulation.

\section{Derivation of sensitivity matrices}

Matrix $\boldsymbol{S}_{P}$ involved in (12) is derived in [7] as:

$$
\boldsymbol{S}_{P}=\boldsymbol{J}_{d c}+\boldsymbol{K}_{V}
$$

where $\boldsymbol{J}_{d c}$ is the Jacobian of DC power injections with respect to DC voltages, and $\boldsymbol{K}_{V}=\operatorname{diag}\left(K_{V 1} \ldots K_{V N}\right)$ is a diagonal matrix with the droop gains as diagonal entries. Matrix $\boldsymbol{S}_{P}$ is nonsingular if there is at least one dispatchable terminal with $K_{V}>0$.

Matrix $S_{I}$ is derived as follows. DC branch currents relate to DC bus voltages through:

$$
\boldsymbol{I}=\boldsymbol{A} \boldsymbol{V}
$$

in which the $l$-th row of $\boldsymbol{A}$ corresponds to the branch between buses $i$ and $m$ and has two nonzero entries:

$$
[\boldsymbol{A}]_{l i}=g_{i m}, \quad[\boldsymbol{A}]_{l m}=-g_{i m}
$$

where $g_{i m}$ is the conductance of the branch under concern.

The linearized behavior is obtained from:

$$
\Delta \boldsymbol{I}=\boldsymbol{A} \Delta \boldsymbol{V}
$$

which, using Eq. (12), can be written as:

$$
\Delta I=A S_{P}^{-1} \Delta P^{s e t}
$$

and hence: $\boldsymbol{S}_{I}=\boldsymbol{A} \boldsymbol{S}_{P}^{-1}$.

Note that the above matrices are small as the number of terminals is not expected to be large.

\section{E. Sensitivity and parameter update after topological changes}

Following a significant event, the behavior of the MTDC grid may change significantly, and the sensitivities and/or reference powers may become outdated. Two cases have to be considered, as detailed next.

1) Tripping of a dispatchable VSC: If the $i$-th VSC has been taken out of service, the droop gain $K_{v, i}$ is set to zero, and matrices $\boldsymbol{S}_{P}$ and $\boldsymbol{S}_{I}$ are recalculated. $\mathbf{S}_{\mathbf{P}}$ is updated by simply subtracting $K_{v, i}$ from the $i$-th diagonal entry.

As far as the Power Rescheduler is concerned, the scheduled power $P_{i}^{s c h}$ and the participation factor $\rho_{i}$ must be set to zero. The participation factors of the remaining VSCs are adjusted accordingly.

This can be illustrated on the example of Fig. 3. Consider the outage of the VSC connected to Area B. Assuming that the zero participation of Area $\mathrm{A}$ is unchanged, the participation factor of Area C becomes $\rho=1$ to satisfy (4). Then, from Eq. (3), the new power references are: $-50 \mathrm{MW}$ for Area A, $0 \mathrm{MW}$ for Area B and $-50 \mathrm{MW}$ for Area C.
2) Tripping of a DC branch: Matrices $\boldsymbol{S}_{P}$ and $\boldsymbol{S}_{I}$ are recalculated by removing the contribution of the tripped branch, and the corresponding constraints (10) are removed. The Power Rescheduler does not need to be updated (unless the HVDC grid splits).

\section{F. Actions on WF terminals}

Some works [10], [23], [24] investigated the possibility of implementing primary DC voltage support by WF converters. Specifically, by adjusting the blade pitch angle, the wind farms can reduce their active power output in order to keep some primary reserves or provide support in case of DC overvoltage. Therefore, they could also be equipped with a droop characteristic. However, this would require dedicated market rules establishing economic incentives to compensate for the long-term sub-optimal operation of the WF. This option is not considered in this paper; instead it is assumed that the WFs are normally operating in MPPT mode. In emergency conditions, however, the controller can send corrective actions to WFs in order to reduce their output temporarily. These actions are to be considered in case of an emergency, thus, the deviation of WF terminals from their reference value is assigned a higher cost $w_{N D}$ in the objective function (6).

Specifically for WF terminals, after the emergency cause has been dealt with, they should return to MPPT operation. To do so, the controller has a "reset mechanism", inspired by the method proposed in [25]. Namely, Eq. (2) is modified as follows for WF terminal $i\left(j=1, \ldots, N_{c}\right)$ :

$$
P_{i}^{r e f}(k+j)=P_{i}^{m}(k)-\frac{j}{N_{c}} \Delta P_{i}^{c o r}(k)
$$

where $\Delta P_{i}^{\text {cor }}(k)$ is the cumulated correction that has been sent to terminal $i$ up to time $k$, i.e.:

$$
\Delta P_{i}^{c o r}(k)=\sum_{j=-\infty}^{k-1} \Delta P_{i}^{s e t}(j)
$$

Clearly, $\Delta P_{i}^{c o r}(k)$ cannot be positive, i.e. the MPC cannot force the $\mathrm{WF}$ to produce more than the maximum power received from wind. If it is equal to zero, then Eq. (24) is the same as Eq. (2). Otherwise, Eq. (24) gives an estimate of the MPPT WF power, if no correction had been sent. The proposed controller will aim to restore the WF to its maximum available power once the constraint necessitating WF curtailment is no longer active.

\section{Simulation RESUlts}

\section{A. Test system}

The proposed control scheme has been tested on a system consisting of two asynchronous AC areas and one offshore wind farm, connected through a five-terminal HVDC grid, as sketched in Fig. 4. A variant of this AC/DC system can be found in [26].

Each AC area is based on the so-called Nordic test system shown in Fig. 5, set up by an IEEE Task Force and detailed in [27]. In both replicas, generator g20, which represented a large external AC system has been removed and the nearby 


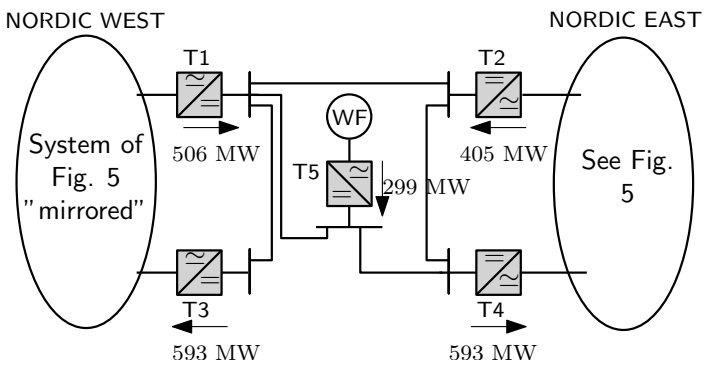

Fig. 4. Test system topology and initial power flow

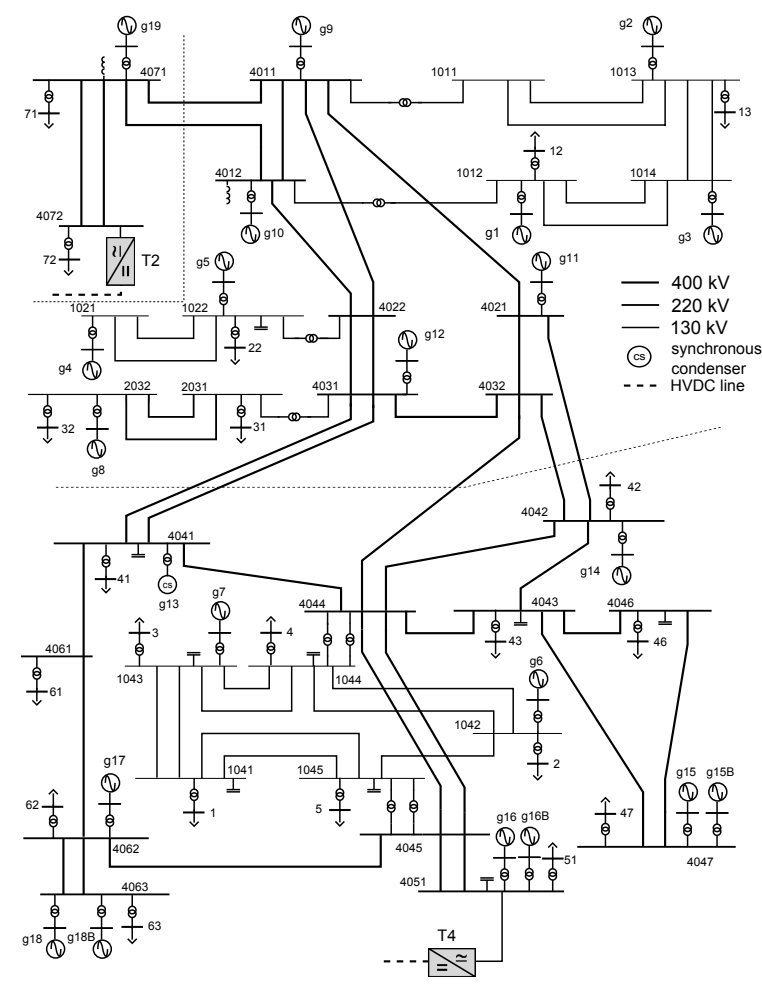

Fig. 5. Nordic East test system with MTDC grid connections

equivalent load has been accordingly adjusted. Each subsystem has two points of connection to the MTDC grid.

All generators are represented with their automatic voltage regulators, excitation systems, speed governors and turbines as detailed in [27]. Each VSC is modeled in some detail with 28 differential-algebraic equations involving the phase reactor, inner and outer control loops, PLLs, filters, etc. (model Type 6 in [28]). The DC branches are represented only by their series resistance by neglecting the series inductance and accounting for their DC capacitances in the terminal capacitors [26].

Among the five VSCs, all but T5 operate in DC voltage droop mode with $K_{v}=5 \mathrm{pu}$ (on the VSC nominal power base). The initial setpoints $P^{\text {set }}$ and $V^{\text {set }}$ for each VSC are set equal to its initial DC power and voltage. T5 imposes constant frequency and voltage on its $\mathrm{AC}$ side, thus acting as a slack bus for the offshore wind farm, merely modeled as a power injection. The initial power in each VSC is shown in Fig. 4.

A control horizon of $N_{c}=3$ steps has been considered for the MPC with a sampling time $T=5 \mathrm{~s}$. This choice is justified in Appendix B. The control actions are applied $1 \mathrm{~s}$ after receiving the measurements, to account for the time needed to process them, solve the optimization problem and communicate corrections to the VSCs. The weighting factors $w_{D}, w_{N D}, q$ and $v$ in the objective function (6) are taken equal to $1,10^{2}, 10^{3}$ and $10^{6}$, respectively. These values have been set based on the importance of each variable, and no further fine-tuning has been contemplated. The same participation factor $\rho=0.25$ has been assumed for all four dispatchable VSCs. Since steady state is assumed at the beginning of the simulation, $V_{\text {avg }}^{r e f}$ is taken equal to the average of the initial DC voltages $(1 \mathrm{pu})$.

For all VSCs, the active power limits have been set to $P^{\min }=-10$ and $P^{\max }=+10$ pu (on a $100 \mathrm{MW}$ base), the DC voltage limits to $V^{\min }=0.96$ and $V^{\max }=1.04$ pu. For simplicity, the rate of change limits have been set to large values $\left(\Delta P^{\min }=-10 \mathrm{pu}\right.$ and $\left.\Delta P^{\max }=+10 \mathrm{pu}\right)$, and were not considered in the simulations. The DC branch current limits have been set to $I^{\max }=10 \mathrm{pu}$, except for both branches connected to T5, which have a lower capacity with $I^{\max }=3.5 \mathrm{pu}$. $V^{\min }, V^{\max }$ and $I^{\max }$ are defined in Appendix A.

\section{B. Scenario 1: WF power decrease and change in schedule}

This first scenario includes the following events:

- from $t=11$ to $t=31 \mathrm{~s}$ : gradual (although accelerated) decrease of the WF power from 299 to $100 \mathrm{MW}$;

- at $t=51 \mathrm{~s}$ : update of the scheduled powers $P_{i}^{s c h}$ of all dispatchable VSCs. The new schedule, corresponding to the new WF production, is as follows: $500 \mathrm{MW}$ in T1, $600 \mathrm{MW}$ in $\mathrm{T} 2,-800 \mathrm{MW}$ in $\mathrm{T} 3$, and $-400 \mathrm{MW}$ in $\mathrm{T} 4$.

Figure 6 shows the DC powers of the converters. As expected, following the WF power decrease, all converters inject more power in the MTDC grid. The corrections calculated and sent by the MPC controller are shown in Fig. 7. They start at $t=15 \mathrm{~s}$. Since all dispatchable terminals have the same participation factor $\rho_{i}$, the system is steered to an operating point where all VSCs are asked to cover the same fraction of the lost WF power.

Following the schedule change at $t=51 \mathrm{~s}$, the VSC powers are smoothly adjusted to follow their new references obtained from Eq. (3). Since the latter neglects MTDC grid losses, the VSC powers do not reach their reference values exactly. The final discrepancies $P-P^{r e f}$ are: $6.4 \mathrm{MW}$ for T1, 6.1 MW for $\mathrm{T} 2,6.7 \mathrm{MW}$ for $\mathrm{T} 3$, and $6.3 \mathrm{MW}$ for $\mathrm{T} 4$. Their sum (25.5 MW) is the total DC power lost. They are (almost) equally distributed over the four VSCs because they were given the same weight $w_{D}$ in the objective function (6).

The DC voltages and their average $V_{\text {avg }}$ are shown in Fig. 8. The decrease of the WF power causes a temporary power deficit in the MTDC grid, which is temporarily covered by the discharging of the DC capacitors, leading to the drop of the DC voltages. The magnitude of the DC voltage drop is determined by the droop gains of the VSCs. However, the centralized controller smoothly restores the average voltage to its reference value ( $1 \mathrm{pu})$, and all voltages eventually settle near this value. 


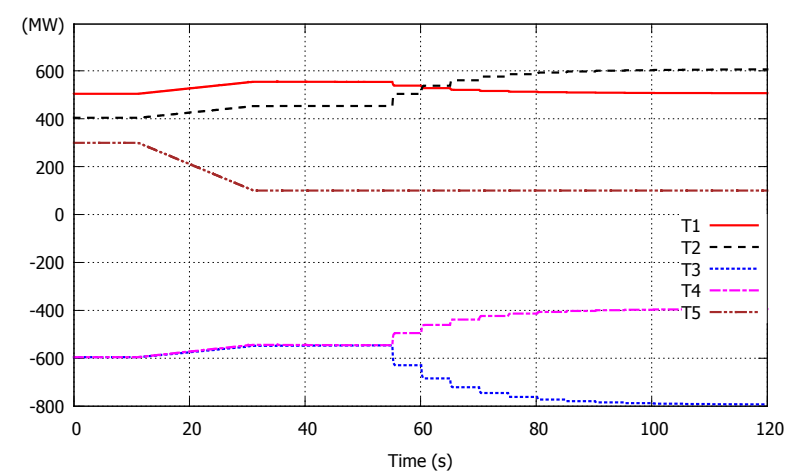

Fig. 6. Scenario 1: DC powers of VSCs

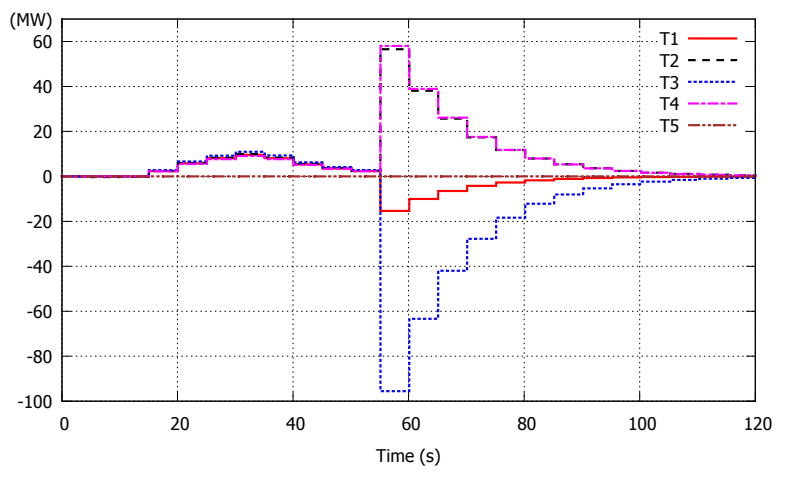

Fig. 7. Scenario 1: setpoint changes $\Delta \boldsymbol{P}^{\text {set }}$

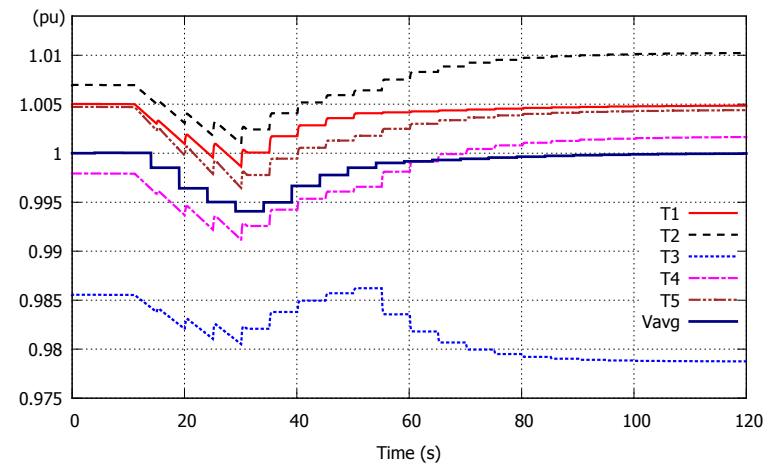

Fig. 8. Scenario 1: MTDC grid voltages

The values of $P^{s c h}$ of the VSCs at various time instants and the values $P^{r e f}$ as calculated by Eqs. (2) and (3) are shown in Table I (rounded to the nearest integer). It is noted that the initial $P^{s c h}$ values are taken equal to the initial DC powers of the VSCs.

\section{Scenario 2: outage of an AC/DC terminal}

This scenario involves the inadvertent tripping of Terminal $\mathrm{T} 3$ at $t=5 \mathrm{~s}$.

Following this outage, the power references $\boldsymbol{P}^{\text {ref }}$ are updated by the Power Rescheduler. As explained in subsection III-E, the scheduled power of T3 and its participation factor are set to zero, while the participation factors of the remaining dispatchable VSCs are adjusted. Several options can be thought of for this adjustment. As in [10], it was assumed that, after the tripping of a VSC connected to an AC area, the power transfer to that area is taken over by the remaining
TABLE I

$P^{s c h}$ AND $P^{r e f}$ VAlues at VARIOUS Time instants (MW)

\begin{tabular}{c|c|ccccc}
\hline Time & & T1 & T2 & T3 & T4 & T5 \\
\hline \multirow{2}{*}{$t=4 \mathrm{~s}$} & $P^{\text {sch }}$ & 506 & 405 & -593 & -593 & - \\
& $P^{r e f}$ & 500 & 400 & -600 & -600 & 299 \\
\hline$t=19 \mathrm{~s}$ & $P^{\text {sch }}$ & 506 & 405 & -593 & -593 & - \\
& $P^{r e f}$ & 520 & 419 & -580 & -580 & 220 \\
\hline$t=34 \mathrm{~s}$ & $P^{s c h}$ & 506 & 405 & -593 & -593 & - \\
& $P^{r e f}$ & 550 & 449 & -549 & -549 & 100 \\
\hline$t=54 \mathrm{~s}$ & $P^{\text {sch }}$ & 500 & 600 & -800 & -400 & - \\
& $P^{r e f}$ & 500 & 600 & -800 & -400 & 100 \\
\hline
\end{tabular}

VSCs connected to it. This leads to setting, once the loss of $\mathrm{T} 3$ is known, $\rho_{1}=1$ for $\mathrm{T} 1$, and $\rho_{2}=\rho_{4}=0$ for $\mathrm{T} 2$ and $\mathrm{T} 4$.

The VSC powers are shown in Fig. 9. Immediately after the outage of T3, thanks to voltage droop control, T1, T2 and T4 adjust rapidly their power to restore the power balance of the MTDC grid. As can be seen in Fig. 10, the controller starts acting at $t=10 \mathrm{~s}$. It redirects the lost power to $\mathrm{T} 1$, with the result that the powers of $\mathrm{T} 2$ and $\mathrm{T} 4$ progressively return to their pre-disturbance values (see Fig. 9).

The corresponding DC voltage evolutions are shown in Fig. 11. Since T3 was operating as inverter before the disturbance, the DC voltages rise rapidly after its tripping, but are stabilized by the DC voltage droop control. However, this alone is not satisfactory, since the DC voltages exceed the allowed upper limit of $1.04 \mathrm{pu}$, and the average DC voltage has drifted from its reference value. Both issues are corrected by the controller smoothly bringing the DC voltages in the specified range and the average voltage to its reference. ${ }^{1}$ Without the centralized control, the DC voltages would remain at the values immediately after the disturbance and the violations observed at some buses would not be corrected.

While the powers are satisfactorily rerouted in the minute that follows this severe disturbance, the modified $\rho_{i}$ values may not be acceptable over a longer period of time. It is the Power Rescheduler role to subsequently modify the power schedule and the participations.

Simulation results in the presence of modeling errors are provided in Table II.

The first two lines of the table show the final operating points reached when using MPC, respectively without and with model inaccuracy. The latter consist of assuming erroneous entries in the $\boldsymbol{K}_{V}$ matrix used by the controller. Namely, $K_{v}=10 \mathrm{pu}$ has been assumed for T1 and T2, instead of the actual $K_{v}=5$ pu value used in the VSC model. Comparing the first two rows of the table shows that the MPC-based controller brought the system to the same final operating point, thus compensating for the unknown error. It is noted that due to the change of the MTDC grid losses, the steady-state power of $\mathrm{T} 1$ will not correspond exactly to the difference of the predisturbance powers of $\mathrm{T} 1$ and $\mathrm{T} 3$.

\footnotetext{
${ }^{1}$ In this figure (and similar ones), the actual DC voltage evolutions are shown, while the average voltage is the one computed by the controller from the received measurements. This explains the (four second) lag that affects the curve of the average voltage.
} 


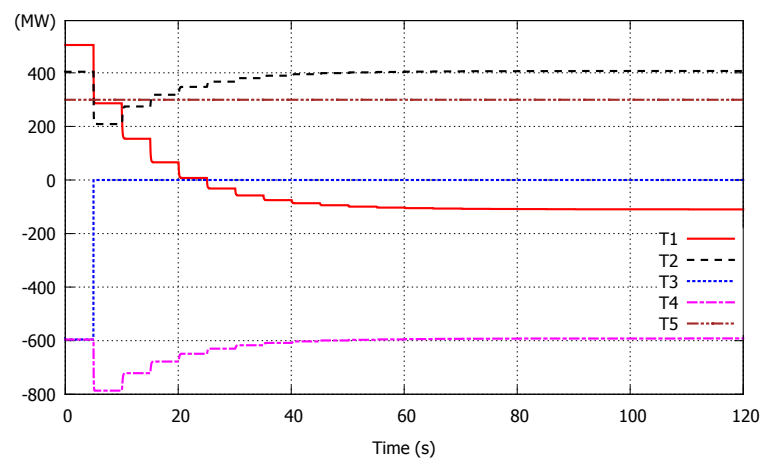

Fig. 9. Scenario 2: DC powers of VSCs

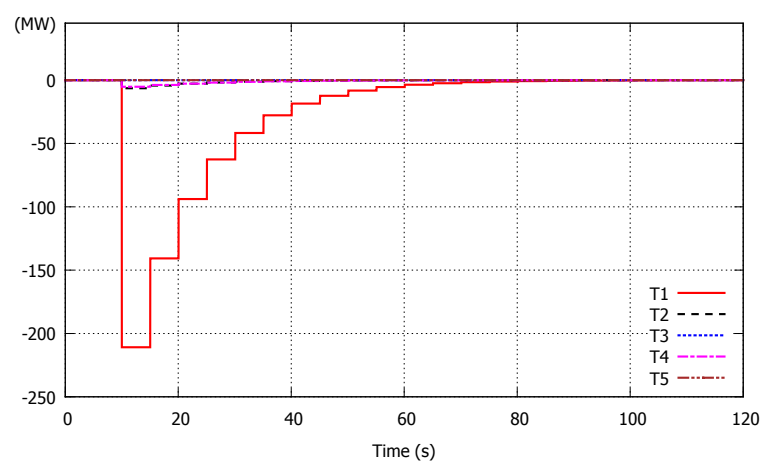

Fig. 10. Scenario 2: setpoint changes $\Delta \boldsymbol{P}^{\text {set }}$

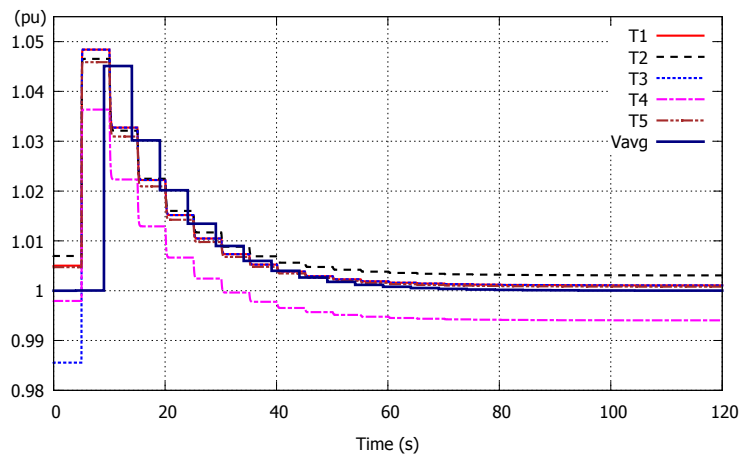

Fig. 11. Scenario 2: MTDC grid voltages

The last two rows of the table relate to an optimizationbased correction method acting in open loop. The latter simply consists of a single-step $\left(N_{c}=1\right)$ minimization of the objective (6) under the constraints (7)-(15). ${ }^{2}$ The so-obtained corrections are applied only once, i.e. without exploiting successively updated measurements as MPC does. With an accurate model, this open-loop correction yields almost the same operating point, showing negligible differences compared to the MPC solution (first two rows). However, if the $\boldsymbol{K}_{V}$ matrix is affected by the aforementioned errors, the resulting operating point is significantly impacted, as shown by the last row in Table II. More precisely, T1 does not compensate for the loss of T3 as expected, and the effective average voltage deviates from its setpoint.

\footnotetext{
${ }^{2}$ Except constraint (9), which has been removed as it is specific to multistep control
}

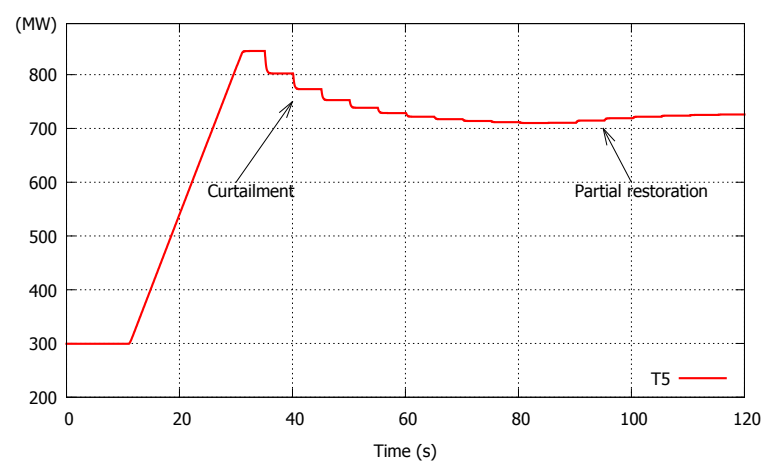

Fig. 12. Scenario 3: DC power of VSC T5

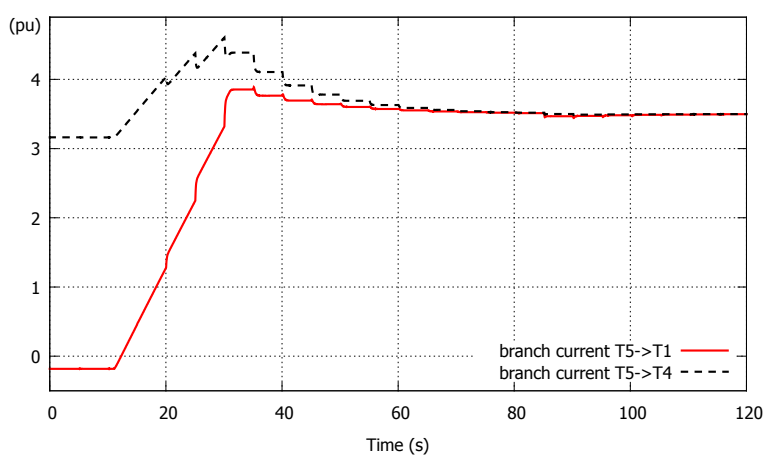

Fig. 13. Scenario 3: Branch currents

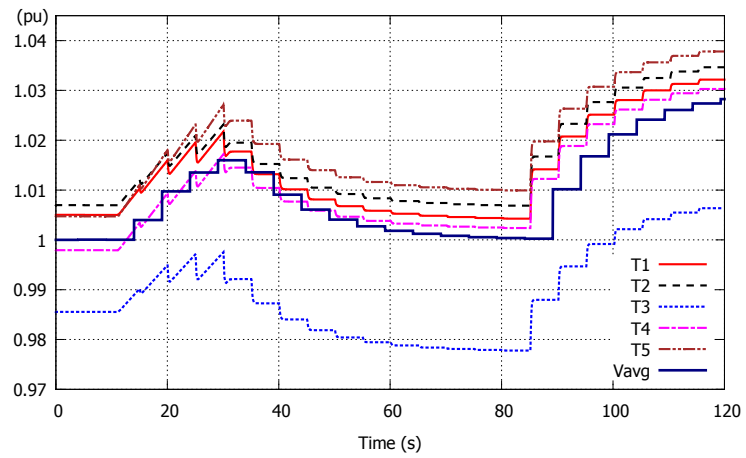

Fig. 14. Scenario 3: MTDC grid voltages

TABLE II

SCENARIO 2 - FINAL OPERATING POINTS WITH VARIOUS METHODS

\begin{tabular}{c|ccc|c}
\hline \multirow{2}{*}{ Method } & \multicolumn{3}{|c|}{ VSC Power (MW) } & $V_{\text {avg }}$ \\
& $\mathrm{T} 1$ & $\mathrm{~T} 2$ & $\mathrm{~T} 4$ & (pu) \\
\hline MPC \& accurate model & -110 & 407 & -592 & 1.00 \\
MPC \& inaccurate model & -110 & 407 & -592 & 1.00 \\
\hline Open-loop \& accurate model & -110 & -406 & -590 & 1.00 \\
Open-loop \& inaccurate model & -192 & -343 & -447 & 0.97 \\
\hline
\end{tabular}

\section{Scenario 3 - Branch overload alleviation}

The last scenario demonstrates the efficiency of the proposed control in correcting branch current violations. As the controller acts on the non-dispatchable terminal T5, it also illustrates the reset mechanism described in sub-section III-F.

The following events are involved:

- from $t=11$ to $t=31 \mathrm{~s}$ : large, gradual (although accelerated) increase of the WF power by $550 \mathrm{MW}$; 
- at $t=80 \mathrm{~s}$ : the average voltage reference $V_{a v g}^{r e f}$ is increased from 1 to $1.03 \mathrm{pu}$. This change could emanate from a higher (tertiary) level of control.

Figure 12 shows the power injected in the grid by T5. It can be seen that at $t=35 \mathrm{~s}$, a fraction of this power is curtailed by the controller. This curtailment is dictated by the overload of the two branches connected to T5, as can be seen in Fig. 13. The limit of branch T5-T4 is the first to be exceeded, and the controller redirects the power injected by T5 through branch T5-T1, which avoids curtailing the WF power. However, after $t=30 \mathrm{~s}$ both branches have their limits exceeded and the controller starts curtailing the WF power. Eventually, some $140 \mathrm{MW}$ are curtailed and the branch currents are brought back below their limits at $t=80 \mathrm{~s}$. At the same time, the DC voltages are adjusted so that their average is brought to $1 \mathrm{pu}$, as can be seen from Fig. 14.

Following the change of the $V_{a v g}^{r e f}$ reference at $t=80 \mathrm{~s}$, the controller increases all the DC voltages. As a result, the DC currents decrease slightly and temporarily, and their respective constraints are no longer active. Thus, the MPC scheme identifies that some more WF power can be accommodated, and it increases the power of T5. This restores a small part of the WF power previously curtailed, until the DC branch current constraints become active again.

\section{CONClusion}

A centralized control scheme has been proposed for the supervisory control of MTDC grids, with a sampling period in the order of a few seconds.

The controller relies on the MPC concept and involves solving a constrained optimization problem at each discrete time. The MPC formulation was chosen for its ability to accommodate model simplifications and uncertainties while incorporating constraints. This allows predicting the system evolution with a static, sensitivity-based model.

The multi-time-step objective consists in tracking power references, while preventing or correcting limit violations.

Those reference values are adjusted by a Power Rescheduler taking care of the power balance of the whole MTDC grid. A simple version of the latter has been considered, using participation factors and neglecting the MTDC grid losses. Procedures have been proposed to update the references and/or participations after a disturbance, and to reset WFs to their available power as soon as possible after a curtailment.

The performance of the controller has been successfully demonstrated through detailed time simulations of a test system including a five-terminal DC grid, in various scenarios including severe terminal outages.

Extensions of the controller may deal with the adjustment of the reference value of the average DC voltage, e.g. to minimize the losses for a range of operating points, while still ensuring the N-1 security of the DC grid. Extensions of the Power Rescheduling procedures are also envisaged.

\section{APPENDIX A}

\section{PRogRESSIVE BOUND TIGHTENING}

In normal operation, the DC voltages lie between the specified minimum $\boldsymbol{V}^{\min }$ and maximum $\boldsymbol{V}^{\max }$ values, and

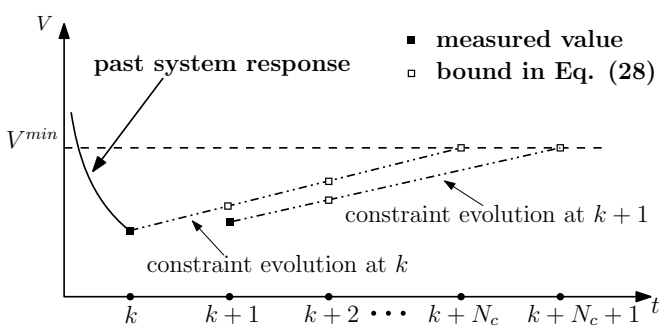

Fig. 15. DC Voltage progressive constraint tightening

the branch currents are below their limits $\boldsymbol{I}^{\max }$. In this case, the bounds in constraints (7) and (10) are $\left(j=1, \ldots, N_{c}\right)$ :

$$
\begin{array}{ll}
\boldsymbol{V}^{\text {low }}(k+j)=\boldsymbol{V}^{\text {min }} & \boldsymbol{V}^{u p}(k+j)=\boldsymbol{V}^{\text {max }} \\
\boldsymbol{I}^{\text {low }}(k+j)=-\boldsymbol{I}^{\text {max }} & \boldsymbol{I}^{\text {up }}(k+j)=\boldsymbol{I}^{\text {max }} .
\end{array}
$$

If, after a disturbance, a terminal DC voltage or a branch current exceeds its limit, the controller should alleviate this violation before it leads to equipment tripping or damage. On the other hand, abrupt corrections should be avoided. Therefore, after a violation is identified, the bounds in (7) and (10) are progressively tightened. More precisely, if the DC voltage $V_{i}^{m}$ measured at terminal $i$ violates its lower limit $V_{i}^{\text {min }}$, the latter is progressively enforced by specifying $\left(j=1, \ldots, N_{c}\right)$ :

$$
V_{i}^{\text {low }}(k+j)=V_{i}^{m}(k)+\frac{j}{N_{c}}\left(V_{i}^{\text {min }}-V_{i}^{m}(k)\right)
$$

This is shown in Fig. 15. The leftmost dash-dotted line is the linearly evolving $V_{i}^{\text {low }}$, considered at time $k$, reaching $V_{i}^{\text {min }}$ at the end of the control horizon, at time $k+N_{c}$. After a new measurement is collected, at time $k+1$, the bound is updated according to the rightmost dash-dotted line.

Of course similar expressions hold for the upper voltage limit and for the current limits as well.

In this paper, a linear function has been chosen for the progressive constraint tightening. Other functions, e.g. exponential, could be also used, if faster correction is required.

This way of alleviating the violations assumes that thermal limits can be exceeded for some time. In HVDC systems, however, some violations may not be tolerated at all. For example, the DC voltage limits have to be chosen with a security margin from the real technical limits because a DC voltage outside limits may cause immediate tripping of the converter. In fact, the soonest the controller can alleviate a violation is after a delay of one time step. For faster reaction, other methods (relying only on local information, or eventbased schemes) should be considered.

\section{APPENDIX B}

\section{SELECTION OF CONTROL HORIZON}

The control horizon is chosen to obtain a desired $5 \%$ settling time. The latter can be easily calculated by neglecting the losses of the MTDC grid and assuming no voltage or current limit is reached. From Eq. (5) the VSC powers after one control step of the MPC will be:

$$
\boldsymbol{P}(k+1)=\boldsymbol{P}(k)+\frac{\boldsymbol{P}^{r e f}-\boldsymbol{P}(k)}{N_{c}}
$$


Therefore, the error $\delta \boldsymbol{P}$ after one step will be:

$$
\delta \boldsymbol{P}(k+1)=\boldsymbol{P}^{r e f}-\boldsymbol{P}(k+1)=\frac{N_{c}-1}{N_{c}}\left(\boldsymbol{P}^{r e f}-\boldsymbol{P}(k)\right)
$$

Following the same logic for the next steps, the error at the $n$-th step will be:

$$
\delta \boldsymbol{P}(k+n)=\left(\frac{N_{c}-1}{N_{c}}\right)^{n}\left(\boldsymbol{P}^{r e f}-\boldsymbol{P}(k)\right)
$$

A settling time of $40 \mathrm{~s}$ has been chosen, which translates to a total of 8 steps for the chosen sampling time of $5 \mathrm{~s}$. Therefore, by substituting $n=8$ in Eq. (31) the maximum control horizon $N_{c}$ is found as follows:

$$
\left(\frac{N_{c}-1}{N_{c}}\right)^{8} \leq 0.05 \Rightarrow N_{c}^{\max }=3 \text { steps. }
$$

\section{REFERENCES}

[1] D. Van Hertem and M. Ghandhari, "Multi-terminal VSC HVDC for the European supergrid: Obstacles," Renewable and Sustainable Energy Reviews, vol. 14, pp. 3156-3163, dec 2010.

[2] C. Dierckxsens, K. Srivastava, M. Reza, S. Cole, J. Beerten, and R. Belmans, "A distributed DC voltage control method for VSC MTDC systems," Electric Power Systems Research, vol. 82, pp. 54-58, jan 2012.

[3] M. Aragüés-Peñalba, A. Egea-Àlvarez, S. G. Arellano, and O. GomisBellmunt, "Droop control for loss minimization in HVDC multi-terminal transmission systems for large offshore wind farms," Electric Power Systems Research, vol. 112, pp. 48-55, 2014.

[4] C. Gavriluta, I. Candela, A. Luna, A. Gomez-Exposito, and P. Rodriguez, "Hierarchical Control of HV-MTDC Systems With Droop-Based Primary and OPF-Based Secondary," IEEE Transactions on Smart Grid, vol. 6, pp. 1502-1510, may 2015.

[5] S. Nanou, O. Tzortzopoulos, and S. Papathanassiou, "Evaluation of an enhanced power dispatch control scheme for multi-terminal HVDC grids using Monte-Carlo simulation," Electric Power Systems Research, 2016.

[6] P. Rault, Dynamic Modeling and Control of Multi-Terminal HVDC Grids. PhD thesis, Ecole Centrale de Lille, L2EP, 2014.

[7] T. M. Haileselassie, Control, Dynamics and Operation of Multi-terminal VSC-HVDC Transmission Systems. PhD thesis, Norwegian University of Science and Technology, 2012.

[8] T. M. Haileselassie, A. G. Endegnanew, and K. Uhlen, "Secondary control in multi-terminal VSC-HVDC transmission system," in Proc. IEEE PES General Meeting, 2015.

[9] J. Beerten and D. Van Hertem, "Analysis of power redispatch schemes for HVDC grid secondary voltage control," in Proc. IEEE PES General Meeting, 2015.

[10] A. Egea-Alvarez, J. Beerten, D. Van Hertem, and O. Gomis-Bellmunt, "Hierarchical power control of multiterminal HVDC grids," Electric Power Systems Research, vol. 121, pp. 1-7, apr 2015.

[11] R. Teixeira Pinto, Multi-Terminal DC Networks System Integration, Dynamics and Control. PhD thesis, Delft University of Tech., 2014.

[12] B. Berggren, K. Linden, and R. Majumder, "DC Grid Control Through the Pilot Voltage Droop Concept - Methods for Establishing Set-Point Tracking," in Proc. IEEE International Energy Conference, 2014.

[13] J. Zhu, C. D. Booth, G. P. Adam, and A. J. Roscoe, "Coordinated direct current matching control strategy for multi-terminal DC transmission systems with integrated wind farms," Electric Power Systems Research, vol. 124, pp. 55-64, 2015.

[14] J. M. Maciejowski, Predictive control: with constraints. Pearson education, 2002.

[15] S. J. Qin and T. A. Badgwell, "A survey of industrial model predictive control technology," Control Eng. Practice, pp. 733-764, 2003.

[16] S. Mariethoz, A. Fuchs, and M. Morari, "A VSC-HVDC Decentralized Model Predictive Control Scheme for Fast Power Tracking," IEEE Transactions on Power Delivery, vol. 29, pp. 462-471, feb 2014.

[17] A. Fuchs, M. Imhof, T. Demiray, and M. Morari, "Stabilization of Large Power Systems Using VSC-HVDC and Model Predictive Control," IEEE Transactions on Power Delivery, vol. 29, pp. 480-488, feb 2014.

[18] P. Mc Namara, R. R. Negenborn, B. De Schutter, G. Lightbody, and S. McLoone, "Distributed MPC for frequency regulation in multiterminal HVDC grids," Control Eng. Practice, pp. 176-187, 2016.
[19] M. J. Carrizosa, Hierarchical control scheme for multi-terminal high voltage direct current power networks. $\mathrm{PhD}$ thesis, Universite Paris Sud, 2015.

[20] S. Cole and R. Belmans, "A proposal for standard VSC HVDC dynamic models in power system stability studies," Electric Power Systems Research, vol. 81, pp. 967-973, apr 2011.

[21] C. Feltes and I. Erlich, "Variable Frequency Operation of DFIG based Wind Farms connected to the Grid through VSC-HVDC Link," in Proc. IEEE PES General Meeting, 2007.

[22] B. Silva, C. L. Moreira, L. Seca, Y. Phulpin, and J. Pecas Lopes, "Provision of Inertial and Primary Frequency Control Services Using Offshore Multiterminal HVDC Networks," IEEE Transactions on Sustainable Energy, vol. 3, pp. 800-808, oct 2012.

[23] B. M. Amaral Silva, Multi-terminal HVDC Grids : Control Strategies for Ancillary Services Provision in Interconnected Transmission Systems with Offshore Wind Farms. PhD thesis, University of Porto, 2013.

[24] I. Martinez-Sanz, Control of AC / DC Systems for Improved Transient Stability and Frequency Support Provision. $\mathrm{PhD}$ thesis, Imperial College London, 2015.

[25] H. Soleimani Bidgoli, M. Glavic, and T. Van Cutsem, "RecedingHorizon Control of Distributed Generation to Correct Voltage or Thermal Violations and Track Desired Schedules," in Proc. 19th IEEE Power Systems Computation Conference, 2016.

[26] L. Papangelis, X. Guillaud, and T. Van Cutsem, "Frequency support among asynchronous AC systems through VSCs emulating power plants," in Proc. IET Intern. Conf. on AC and DC Power Transm., 2015.

[27] IEEE PES Task Force, "Test Systems for Voltage Stability Analysis and Security Assessment," tech. rep., 2015. Available online on IEEE PES Resource Center.

[28] CIGRE WG B4.57, Guide for the Development of Models for HVDC Converters in a HVDC Grid. CIGRE Technical Brochure 604.

Lampros Papangelis (S'14) received the Diploma degree in Electrical and Computer Engineering from the National Technical University of Athens, Greece, in 2013 . He is currently pursuing the Ph.D. degree at the University of Liège. His research interests include the modeling and control of AC/DC systems, in particular MTDC grids.

Marie-Sophie Debry graduated from the Ecole Supérieure d'Electricité (Supelec) in 2005. She has since been working in the Power System Expertise dept. of the French transmission system operator RTE. She was involved in several EU-funded project (EWIS, e-Highway2050) as well as in the FranceSpain HVDC link project. As part of the Horizon 2020 Migrate project, she is responsible of a work package enabling a transition towards HVAC systems where all generation and consumption is connected via power electronics.

Patrick Panciatici (SM'06) graduated from Ecole Supérieure d'Electricité (Supelec), France. He joined EDF R\&D in 1985, and RTE in 2003, where he is scientific advisor in the R\&D direction. He coordinates and supervises long-term research activities, in particular large projects supported by EU (PEGASE, Twenties, iTesla, e-HIGHWAY2050, Migrate, etc.). He is a member of CIGRE and senior member of IEEE and SEE (France). He is the representative of RTE in PSERC (Power Systems Engineering Research Center), USA.

Thierry Van Cutsem (F' 05) received the M.Sc. and Ph.D. degrees from the University of Liège, Belgium, in 1979 and 1984, respectively. He is currently a Research Director of the Fund for Scientific Research (FNRS) and an Adjunct Professor at the Dept. of Electrical Engineering and Computer Science at the same university. His research interests include power system dynamics, security, monitoring, control, and simulation. He is currently the Past Chair of the IEEE PES Power System Dynamic Performance Committee. 